Images in clinical medicine

\title{
An unusual communication between the trunk of the mandibular nerve and the lingual nerve in a female cadaver
}

Sitthichai Iamsaard*, Jeerapat Singsorn, Porntip Boonruangsri

Department of Anatomy, Faculty of Medicine, Khon Kaen University, Khon Kaen, Thailand
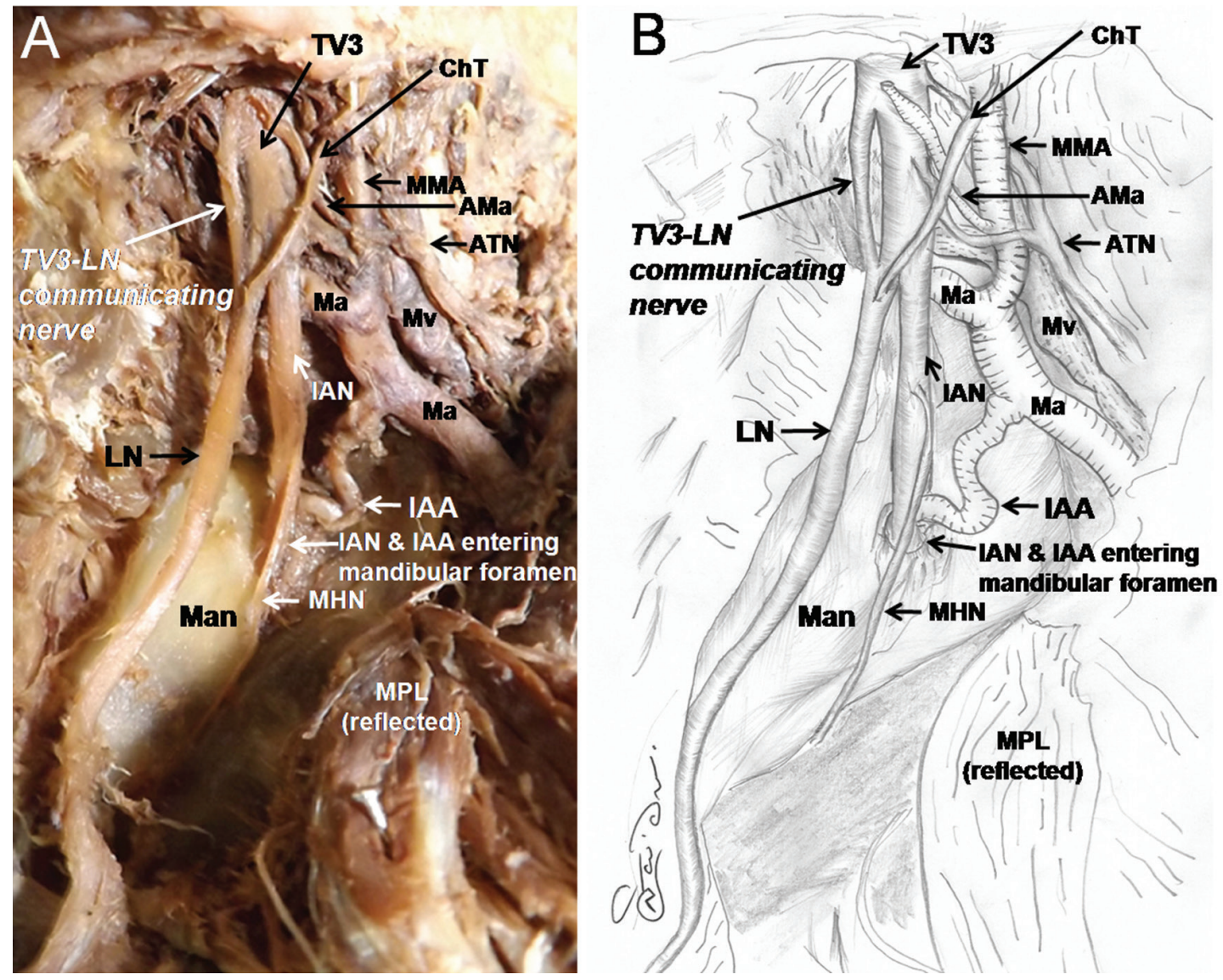

Figure $1 \mathrm{~A}$ photograph (A) and an illustration (B) showing the left-medial aspect of the unusual communication between trunk of mandibular nerve (TV3) and the lingual nerve (LN) communication in a 71 year old woman. ChT: chorda tympani nerve; MMA: middle meningeal artery; AMa: accessory meningeal artery; ATN: auriculotemporal nerve; Ma: maxillary artery; Mv: maxillary vein; IAA: inferior alveolar artery; IAN: inferior alveolar nerve; MHN: mylohyoid nerve; MPT: medial pterygoid muscle; Man: mandible. 
In general, TV 3 emits 6 branches from the anterior and posterior division of its trunk. Branches of these divisions supply the meninges, the tensor tympani and tensor veli palatini muscles, the muscles of mastication, the anterior belly of digastric and mylohyoid muscles, the salivary glands, teeth and gingivae, and the maxillary sinus including the sensory innervation of the face. Anomalous communications among the branches of the TV3 have been clinically documented in Koreans (1), Turks (2), and Indians (3) because they can possibly explain the unsuccessful anesthesia before surgery in the infratemporal and mandibular region or the oral cavity. Common variations reported are communications of the LN with the MHN or the ATN (1-3). It was also mentioned that the IAN could communicate with the ATN or the LPN (1). Exceptionally, Erdogmus et al. (2) demonstrated an unusual communication between the LN and the MPN. Variant communications (such as the LN to the IAN or the ATN) as previously documented in several populations, may also be observed in Thai cadavers during the gross anatomy dissection. Specifically, from 102 samples (51 heads), we found a very rare and unusual communication between the TV3 and the LN (TV3-LN communication) in a 71 year old Thai woman. This communication is reported for the first time. It seems to be a posterior division of the TV3 that communicates above the junction between the LN and the chorda tympani nerve (Figure 1).
This uniquely anomalous communication should be noted and documented in cases of anesthesia in the mandibular and infratemporal region, or the oral cavity in various treatment procedures.

Key words: Mandibular nerve trunk - Lingual nerve - Nerve communication.

Authors' contributions: Conception and design: SI; Acquisition, analysis and interpretation of data: SI, JS, PB; Drafting the article: SI; Revising it critically for important intellectual content: SI, PB.

Conflict of interest: The authors declare that they have no conflict of interest.

\footnotetext{
${ }^{*}$ Corresponding author sittia@kku.ac.th

Tel.: + 6643363212

Fax.: + 6643363212
}

Received: 1 March 2015; Accepted: 4 August 2015

\section{References}

1. Kim SY, Hu KS, Chung IH, Lee EW, Kim HJ. Topographic anatomy of the lingual nerve and variations in communication pattern of the mandibular nerve branches. Surg Radiol Anat. 2004;26(2):128-35.

2. Erdogmus S, Govsa F, Celik S. Anatomic position of the lingual nerve in the mandibular third molar region as potential risk factors for nerve palsy. J Craniofac Surg. 2008;19(1):264-70.

3. Thotakura B, Rajendran SS, Gnanasundaram V, Subramaniam A. Variations in the posterior division branches of the mandibular nerve in human cadavers. Singapore Med J. 2013;54(3):149-51. 